\title{
Bounds of Operator Functions and Furuta Inequalities
}

\author{
By
}

\author{
Chia-Shiang LIN *
}

\begin{abstract}
In this paper we shall find bounds of operator functions of the Furuta types, and bounds of the monotone decreasing function $\mathrm{F}_{p, t}(A, B, r, s)$ of operators in particular. Consequently, we obtain bounds of Furuta inequalities.
\end{abstract}

\section{§1. Notation and Introduction}

Throughout this note it is to be understood that the capital letters mean bounded linear operators acting on a Hilbert space $\mathrm{H}$. $T$ is positive (written $T \geq O$ ) in case $(T x, x) \geq 0$ for all $x \in$ H. $T>O$ in case $T$ is positive and invertible. If $S$ and $T$ are selfadjoint, we write $T \geq S$ in case $T-S \geq O$. $I$ will denote the identity operator.

First of all, we recall the Furuta inequality which is the origin of the results discussed in this paper.

Theorem $\mathbf{F}$ (Furuta inequality)

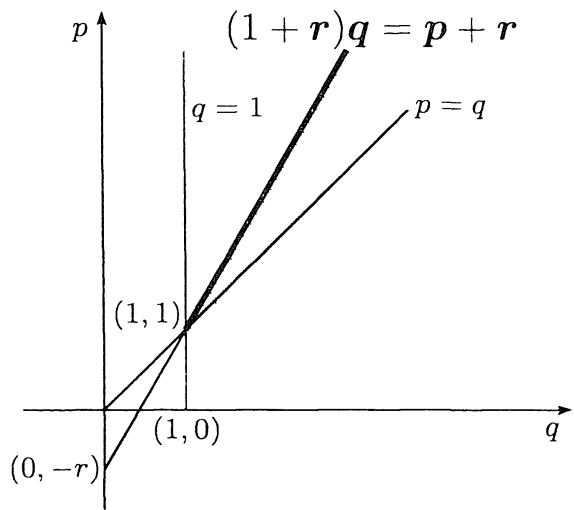

Figure

[1]. If $A \geq B \geq O$, then for each $r \geq 0$,

Communicated by T. Kawai, November 17, 1999. Revised January 13, 2000.

1991 Mathematics Subject Classification(s): 47B15

*Department of Mathematics, Bishop's University, Lennoxville, P.Q. J1M 1Z7, Canada. 


$$
\left(B^{r / 2} A^{p} B^{r / 2}\right)^{1 / q} \geq\left(B^{r / 2} B^{p} B^{r / 2}\right)^{1 / q}
$$

and

$$
\left(A^{r / 2} A^{p} A^{r / 2}\right)^{1 / q} \geq\left(A^{r / 2} B^{p} A^{r / 2}\right)^{1 / q}
$$

hold for $p \geq 0$ and $q \geq 1$ with $(1+r) q \geq p+r$.

An elementary one page proof of Theorem $\mathrm{F}$ appeared in [2], and the domain for $p, q$ and $r$ in the Figure is the best possible for Theorem F, cf. [7] for the proof. We also require the Löwner-Heinz formula which is a special case of Theorem F, i.e., if $A \geq B \geq O$, then $A^{\alpha} \geq B^{\alpha}$ for $\alpha \in[0,1]$; but the inequality does not hold in general for $\alpha>1$.

We shall establish upper and lower bounds of operator functions of the Furuta types in this paper. In fact they are all equivalent to a simple operator inequality. Necessary and sufficient conditions are given for inequalities becoming equalities. Bounds of the monotone decreasing function $\mathrm{F}_{p, t}(A, B, r, s)$ of operators, indicated below, are obtained, and consequently we have bounds of Furuta inequalities.

\section{§2. Bounds of Operator Functions}

Theorem $\mathrm{A}^{\prime}$ in [5] says that if $A \geq B \geq O$ and $A>O$, then for $t \in$ $[0,1], 1 \geq q \geq t \geq 0$ and $p \geq q$ we have the monotone decreasing function $\mathrm{F}_{p . t}(A, B, r, s)$ of operators for both $r \geq t$ and $s \geq 1$, where

$$
\mathrm{F}_{p, t}(A, B, r, s)=A^{-r / 2}\left[A^{r / 2}\left(A^{-t / 2} B^{p} A^{-t / 2}\right)^{s} A^{r / 2}\right]^{\frac{q-t+r}{(p-t) s+r}} A^{-r / 2} .
$$

Moreover (cf. [4] for a simplified proof),

$$
A^{q-t+r} \geq\left[A^{r / 2}\left(A^{-t / 2} B^{p} A^{-t / 2}\right)^{s} A^{r / 2}\right]^{\frac{q-t+r}{(p-t) s+r}} .
$$

In our first result we prove that a simple operator inequality is equivalent to bounds of operator function of Furuta types. It is well-known that the function $f(x)=\lambda x+1-\lambda-x^{\lambda}$ is nonnegative for positive real $x$ and $\lambda \in[0,1]$; and $f(x)$ has the minimum value 0 at $x=1$ by elementary calculus. It follows by the standard operator calculus that we should obtain a simple inequality (1) in Theorem 1 below for $T>O$ and $\lambda \in[0,1]$.

Theorem $\mathbb{1}$. Let $T, A, B>O . t \in[0,1], 1 \geq q \geq t \geq 0, p \geq q, s \geq 1$, and $r \geq t$ such that $(p-t) s+r \neq 0$. Then the following inequalities hold, and they are all equivalent to one another. 


$$
\lambda T+I-\lambda I \geq T^{\lambda} \text { for } \lambda \in[0,1]
$$

$$
\begin{aligned}
& \lambda T+I-\lambda I \leq T^{\lambda} \text { for } \lambda>1 \\
& \lambda T+I-\lambda I \leq T^{\lambda} \text { for } \lambda<0
\end{aligned}
$$

$$
\lambda T+I-\lambda I \geq T^{\lambda} \geq\left(\lambda T^{-1}+I-\lambda I\right)^{-1} \text { for } \lambda \in[0,1]
$$

$$
\lambda T+I-\lambda I \leq T^{\lambda} \leq\left(\lambda T^{-1}+I-\lambda I\right)^{-1} \text { for } \lambda>1
$$

$$
\lambda T+I-\lambda I \leq T^{\lambda} \leq\left(\lambda T^{-1}+I-\lambda I\right)^{-1} \text { for } \lambda<0
$$

(7) $\left[\lambda A^{\frac{r-t}{2}} B^{p} A^{\frac{r-t}{2}}+(I-\lambda I) A^{r}\right]^{\frac{q-t+r}{(p-t) s+r}}$

$$
\begin{aligned}
& \geq\left[A^{r / 2}\left(A^{-t / 2} B^{p} A^{-t / 2}\right)^{\lambda} A^{r / 2}\right]^{\frac{q-t+r}{(p-t) s+r}} \\
& \geq\left[A^{r / 2}\left(\lambda A^{t / 2} B^{-p} A^{t / 2}+I-\lambda I\right)^{-1} A^{r / 2}\right]^{\frac{q-t+r}{(p-t) s+r}} \text { for } \lambda \in[0,1] ;
\end{aligned}
$$

(8) $\quad\left[\lambda A^{\frac{r-t}{2}} B^{p} A^{\frac{r-t}{2}}+(I-\lambda I) A^{r}\right]^{\frac{q-t+r}{(p-t) \propto+1}}$

$$
\begin{aligned}
& \leq\left[A^{r / 2}\left(A^{-t / 2} B^{p} A^{-t / 2}\right)^{\lambda} A^{r / 2}\right]^{\frac{q-t+1}{(p-t) s+r}} \\
& \leq\left[A^{r / 2}\left(\lambda A^{t / 2} B^{-p} A^{t / 2}+I-\lambda I\right)^{-1} A^{r / 2}\right]^{\frac{q-t+r}{(p-t) a+r}} \text { for } \lambda>1 ;
\end{aligned}
$$

(9)

$$
\begin{aligned}
& {\left[\lambda A^{\frac{r-t}{2}} B^{p} A^{\frac{l-t}{2}}+(I-\lambda I) A^{r}\right]^{\frac{q-t+r}{(p-t) a+r}}} \\
& \quad \leq\left[A^{r / 2}\left(A^{-t / 2} B^{p} A^{-t / 2}\right)^{\lambda} A^{r / 2}\right]^{\frac{q-t+r}{(p-t) s+r}} \\
& \quad \leq\left[A^{r / 2}\left(\lambda A^{t / 2} B^{-p} A^{t / 2}+I-\lambda I\right)^{-1} A^{r / 2}\right]^{\frac{q-t+r}{(p-t) \&+r}} \text { for } \lambda<0 .
\end{aligned}
$$

Moreover, equalities hold in (1) and (4) if, and only if $\lambda=0$, or $\lambda=1$, or $T=I$. Equalities hold in (2), (3), (5), and (6) if, and only if $T=I$. Equalities hold in (7) if, and only if $\lambda=0$, or $\lambda=1$, or $B^{p}=A^{t}$. And equalities hold in (8) and (9) if, and only if $B^{p}=A^{t}$.

Proof. (1) holds by a previous remark. That (1), (2) and (3) are all equivalent was proved in [3, Theorem 2]. (4) was mentioned in the proof of $[3$, Theorem 1]. In fact, it follows by taking inverse of the inequality $\lambda T^{-1}+I-\lambda I \geq$ 
$T^{-\lambda}$ which is obtained from (1). Thus, (1) if and only if (4). A similar argument for inequalities (2) and (5), and (3) and (6). So, inequalties (1), (2), (3), (4), (5), and (6) are all equivalent to one another.

Notice that if $S \geq O$, then $S^{\beta} \geq O$ for any real number $\beta$. Let $T=$ $A^{-t / 2} B^{p} A^{-t / 2}>O$ in (4), and then multiply $A^{r / 2}$ on each term from the left and the right as well. Then we have

$$
\begin{aligned}
\lambda A^{\frac{r-t}{2}} & B^{p} A^{\frac{r-t}{2}}+(I-\lambda I) A^{r} \\
& \geq A^{r / 2}\left(A^{-t / 2} B^{p} A^{-t / 2}\right)^{\lambda} A^{r / 2} \\
& \geq A^{r / 2}\left(\lambda A^{t / 2} B^{-p} A^{t / 2}+I-\lambda I\right)^{-1} A^{r / 2} .
\end{aligned}
$$

Now, as $\frac{q-t+r}{(p-t) s+r} \in[0,1]$, by Löwner-Heinz formula we should get the desired inequalities in (7). (8) and (9) can be carried out similarly by using (5) and (6), respectively. Conversely, let $B=T, p=q=s=1$ and $t=0$ in (7), (8) and (9), then we have (4), (5) and (6), respectively. Therefore, the proof of equivalent statements is completed.

Now, suppose that equalities hold in (4), i.e.,

$$
\lambda T+I-\lambda I=\left(\lambda T^{-1}+I-\lambda I\right)^{-1} .
$$

Then, after a straightforwards computation,

$$
\lambda(1-\lambda)\left(T+T^{-1}-2 I\right)=O .
$$

Hence, $\lambda=0$, or $\lambda=1$, or $T-I=O$. The converse is trivial. This applies to equality in (1), too. About equalities in (2), (3), (5), and (6), they can be similarly obtained. Finally, considerations of equalities in (7), (8) and (9) follow from considerations of that in (4), (5) and (6), respectively. Notice that $I=T=A^{-t / 2} B^{p} A^{-t / 2}$ means $B^{p}=A^{t}$. The proof of the theorem is thus completed.

The next result is about bounds of the monotone decreasing operator function $\mathrm{F}_{p, t}(A, B, r, s)$ previously mentioned. We shall omit the proof since it is a special case of (8) in Theorem 1 when $\lambda=s>1$.

Corollary 1. Let $A, B>O, t \in[0,1], 1 \geq q \geq t \geq 0, p \geq q, r \geq t$, and $s>1$. Then

$$
\begin{aligned}
A^{-r / 2} & {\left[s A^{\frac{r-t}{2}} B^{p} A^{\frac{r-t}{2}}+(I-s I) A^{r}\right]^{\frac{q-t+r}{(p-t) s+r}} A^{-r / 2} } \\
& \leq \mathrm{F}_{p . t}(A, B, r, s) \\
& \leq A^{-r / 2}\left[A^{r / 2}\left(s A^{t / 2} B^{-p} A^{t / 2}+I-s I\right)^{-1} A^{r / 2}\right]^{\frac{q-t+r}{(p-t) s+r}} A^{-r / 2}
\end{aligned}
$$

if $(p-t) s+r \neq 0$. Equalities hold if, and only if $B^{p}=A^{t}$. 


\section{§3. Applications-Bounds of Furuta Inequalities}

Notice that in Theorem 1 and Corollary 1 we didn't assume that $A \geq B$, besides $A, B>O$. But it is necessary to assume this condition in this section for an obvious reason. Upper and lower bounds of a Furuta inequality (described in (1) of Lemma below) together with its equivalent inequalities, and in particular the upper and lower bounds of the original Furuta inequality (described in $\left(3^{\prime}\right)$ of Corollary 2 below) together with its equivalent inequalities, will be given in this section. Firstly for completeness sake let us proof the next lemma which appeared in $[6$, Theorem 1$]$.

Lemma. Let $A \geq B \geq O$ with $A>O, 1 \geq q \geq t \geq 0, p \geq q, s \geq 1$, and $r \geq t$ such that $(p-t) s+r \neq 0$. Then the following inequalities hold and are mutually equivalent.

(1) $A^{q-t+r} \geq\left[A^{r / 2}\left(A^{-t / 2} B^{p} A^{-t / 2}\right)^{s} A^{r / 2}\right]^{\frac{q-t+r}{(p-t) s+1}}$ (A Furuta inequality [4]);

$$
\begin{aligned}
A^{\alpha(q-t+r)} \geq\left[A^{r / 2}\left(A^{-t / 2} B^{p} A^{-t / 2}\right)^{s} A^{r / 2}\right]^{\frac{a(q-t+r)}{(p-t) s+r}} \text { for } \alpha \in(0,1] ; \\
A^{\frac{(p-t) s+1}{w}} \geq\left[A^{r / 2}\left(A^{-t / 2} B^{p} A^{-t / 2}\right)^{s} A^{r / 2}\right]^{\frac{1}{w}}
\end{aligned}
$$

for $w \geq 1$ such that $(q-t+r) w \geq(p-t) s+r$.

Proof. The inequality in (1) holds true due to, for instance, [4].

(1) implies (2) by the Löwner-Heinz formula as $\alpha \in(0,1]$.

(2) implies (3). Let $\frac{\alpha(q-t+r)}{(p-t) s+r}=\frac{1}{w}$. Then $\alpha(q-t+r)=\frac{(p-t) s+r}{w}$ and $\frac{q-t+r}{(p-t) s+r} \geq \frac{1}{w}$, so that $(q-t+r) w \geq(p-t) s+r$. But $(p-t) s \geq q-t$, and hence $w \geq 1$. Incidentally, if $0 \leq p, s \leq 1$ and $w \geq 1$, then, by the Löwner-Heinz formula, (3) always holds true.

(3) implies (1). In (3) let $(q-t+r) w=(p-t) s+r$ in particular.

Corollary 2. Let $A \geq B \geq O$ and $r \geq 0$. Then the following inequalities hold and are mutually equivalent.

$$
\begin{gathered}
A^{1+r} \geq\left(A^{r / 2} B^{p} A^{r / 2}\right)^{\frac{1+r}{p+r}} \text { with } p \geq 1 ; \\
A^{\alpha(1+r)} \geq\left(A^{r / 2} B^{p} A^{r / 2}\right)^{\frac{\alpha(1+r)}{p+r}} \text { with } p \geq 1 \text { and } \alpha \in(0,1] ; \\
A^{\frac{p+r}{w}} \geq\left(A^{r / 2} B^{p} A^{r / 2}\right)^{\frac{1}{w}} \text { (The original Furuta inequality [1]) }
\end{gathered}
$$

for $p \geq 0$ and $w \geq 1$ such that $(1+r) w \geq p+r$. 
Proof. This is obviously a consequence of Lemma when $q=s=1$ and $t=0$, and so $p \geq 1$. Notice that $\left(3^{\prime}\right)$ always holds true when $1 \geq p \geq 0$ by the Löwner-Heinz formula, and hence $p \geq 0$.

Now, we are ready to find upper and lower bounds of the three inequalities in Lemma, and, consequently, bounds of the three inequalities in Corollary 2.

Theorem 2. Let $A, B>O, A \geq B, t \in[0,1], 1 \geq q \geq t \geq 0, p \geq q$, $r \geq t, \alpha \in(0,1]$, and $s>1$. Then

(i) $\left[s A^{\frac{r-t}{2}} B^{p} A^{\frac{r-t}{2}}+(I-s I) A^{r}\right]^{\frac{q-t+r}{(p-t) s+r}}$

$$
\begin{aligned}
& \leq\left[A^{r / 2}\left(A^{-t / 2} B^{p} A^{-t / 2}\right)^{s} A^{r / 2}\right]^{\frac{q-t+r}{(p-t) s+r}} \\
& \leq A^{q-t+r} \\
& \leq \begin{cases}{\left[(q-t+r) A^{-1}+(1-q+t-r) I\right]^{-1}} & (q-t+r>1) \\
(q-t+r) A+(1-q+t-r) I & (q-t+r \in[0,1]) .\end{cases}
\end{aligned}
$$

(ii) $\left[s A^{\frac{r-t}{2}} B^{p} A^{\frac{i-t}{2}}+(I-s I) A^{r}\right]^{\frac{a(q-t+r)}{(p-t) s+r}}$

$$
\begin{aligned}
& \leq\left[A^{r / 2}\left(A^{-t / 2} B^{p} A^{-t / 2}\right)^{s} A^{r / 2}\right]^{\frac{\alpha(q-t+r)}{(p-t) s+r}} \\
& \leq A^{\alpha(q-t+r)} \\
& \leq \begin{cases}{\left[\alpha(q-t+r) A^{-1}+(1-\alpha(q-t+r)) I\right]^{-1}} & (\alpha(q-t+r)>1) \\
\alpha(q-t+r) A+(1-\alpha(q-t+r)) I & (\alpha(q-t+r) \in[0,1]) .\end{cases}
\end{aligned}
$$

(iii) $\left[s A^{\frac{r-t}{2}} B^{p} A^{\frac{r-t}{2}}+(I-s I) A^{r}\right]^{\frac{1}{w}}$

$$
\begin{aligned}
& \leq\left[A^{r / 2}\left(A^{-t / 2} B^{p} A^{-t / 2}\right)^{s} A^{r / 2}\right]^{\frac{1}{w}} \\
& \leq A^{\frac{(p-t) s+r}{w}} \\
& \leq \begin{cases}w\left[((p-t) s+r) A^{-1}+(w-((p-t) s+r) I]^{-1}\right. & \left(\frac{(p-t) s+r}{w}>1\right) \\
\frac{1}{w}[((p-t) s+r) A+(w-(p-t) s-r) I] & \left(\frac{(p-t) s+r}{w} \in[0,1]\right)\end{cases}
\end{aligned}
$$

for $w \geq 1$ such that $(q-t+r) w \geq(p-t) s+r$.

Moreover, in all (i), (ii) and (iii) the first inequalities become equalities if, and only if $B^{p}=A^{t}$. In all (i), (ii) and (iii) the last inequalities become equalities if, and only if $A=I$ for each of the first case; and as for the second cases: equality holds in (i) if and only if $q-t+r=0$, or 1 , or $A=I$; equality holds in (ii) if and only if $\alpha(q-t+r)=0$, or 1 , or $A=I$; equality in (iii) holds if and only if $\frac{(p-t) s+r}{w}=0$, or 1 , or $A=I$.

Proof. To show inequalities in (i), the first inequality is obtained from Corollary 1, and the last ones follow from (5) and (4), respectively, in Theorem 
1. By using Lemma, (ii) and (iii) are obtained from (i) above, and (5) and (4), respectively, in Theorem 1.

As for equalities, in all (i), (ii) and (iii) the first inequalities become equalities if, and only if $B^{p}=A^{t}$ due to Corollary 1 , and the last inequalities become equalities due to (5) or (4) in Theorem 1.

Corollary 3. Let $A, B>O, A \geq B, p \geq 1, \alpha \in(0,1]$, and $r \geq 0$. Then

$$
\begin{aligned}
{\left[p A^{r / 2}\right.} & \left.B A^{r / 2}+(I-p I) A^{r}\right]^{\frac{1+1}{p+1}} \\
& \leq\left(A^{r / 2} B^{p} A^{r / 2}\right)^{\frac{1+r}{p+r}} \\
& \leq A^{1+r} \leq\left[(1+r) A^{-1}-r I\right]^{-1}
\end{aligned}
$$

(ii) $\left[p A^{r / 2} B A^{r / 2}+(I-p I) A^{r}\right]^{\frac{a(1+r)}{p+r}}$

$$
\begin{aligned}
& \leq\left(A^{r / 2} B^{p} A^{r / 2}\right)^{\frac{\alpha(1+r)}{p+r}} \\
& \leq A^{\alpha(1+r)} \\
& \leq \begin{cases}{\left[\alpha(1+r) A^{-1}+(1-\alpha(1+r)) I\right]^{-1}} & (\alpha(1+r)>1) \\
\alpha(1+r) A+(1-\alpha(1+r)) I & (\alpha(1+r) \in[0,1]) .\end{cases}
\end{aligned}
$$

$$
\begin{aligned}
{\left[p A^{r / 2}\right.} & \left.B A^{r / 2}+(I-p I) A^{r}\right]^{1 / w} \\
& \leq\left(A^{r / 2} B^{p} A^{r / 2}\right)^{\frac{1}{w}} \\
& \leq A^{\frac{p+r}{w^{\prime}}} \\
& \leq \begin{cases}w\left[(p+r) A^{-1}+(w-p-r) I\right]^{-1} & \left(\frac{p+r}{w}>1\right) \\
\frac{1}{w}[(p+r) A+(w-p-r) I] & \left(\frac{p+r}{w} \in[0,1]\right)\end{cases}
\end{aligned}
$$

for $w>1$ such that $(1+r) w \geq p+r$. In fact the second and third inequalitıes hold for $p \geq 0$.

Moreover, in all (i), (ii) and (iii) the first inequalities become equalities if, and only if $B=I$. In (i) the last inequality becomes equality if and only if $A=I$, or $r=0$. In (ii) and (iii) the last inequalities become equalities if and only if $A=I$ for each of the first case; and as for the second cases: equality holds in (ii) if and only if $\alpha(1+r)=1$, or $A=I$; equality in (iii) holds if and only if $\frac{p+r}{w}=1$, or $A=I$.

Proof. Let $t=0$ and $p=q=1$ in Theorem 2, and then replace $s$ by $p$. Then inequalities in (i), (ii) and (iii) follow immediately. Considerations of equalities are due to special cases of Theorem 2 . 


\section{References}

[1] Furuta, T., $A \geq B \geq O$ assures $\left(B^{r} A^{p} B^{r}\right)^{1 / q} \geq B^{(p+2 r) / q}$ for $r \geq 0, p \geq 0, q \geq 1$ with $(1+2 r) q \geq p+2 r$, Proc. Amer. Math. Soc., 101 (1987), 85-88.

[2] Furuta, T., Elementary proof of an order preserving inequality, Proc. Japan Acad., 65 (1989), 126.

[3] Furuta, T., and Yanagida, M., Generalized means and convexity of inversion for positive operators, Amer. Math. Monthly, 105 (1998), 258-259.

[4] Furuta, T., Simplified proof of an order preserving operator inequality, Proc. Japan Acad., 74 (1998), 114.

[5] Furuta, T., and Wang, D., A decreasing operator function associated with the Furuta inequality, Proc. Amer. Math. Soc., 126 (1998), 2427-2432.

[6] Lin, C.-S., Unifying statement for Furuta inequalities, Preprint.

[7] Tanahashi, K., Best possibility of the Furuta inequality, Proc. Amer. Math. Soc., 124 (1996), 141-146. 